\title{
Flagged Parallel Manipulators
}

\author{
Maria Alberich-Carramiñana, Federico Thomas, Member, IEEE, and Carme Torras, Member, IEEE
}

\begin{abstract}
The conditions for a parallel manipulator to be flagged can be simply expressed in terms of linear dependencies between the coordinates of its leg attachments, both on the base and on the platform. These dependencies permit to describe the manipulator singularities in terms of incidences between two flags (hence, the name "flagged"). Although these linear dependencies might look, at first glance, too restrictive, in this paper, the family of flagged manipulators is shown to contain large subfamilies of six-legged and three-legged manipulators. The main interest of flagged parallel manipulators is that their singularity loci admit a well-behaved decomposition with a unique topology irrespective of the metrics of each particular design. In this paper, this topology is formally derived and all the cells, in the configuration space of the platform, of dimension 6 (nonsingular) and dimension 5 (singular), together with their adjacencies, are worked out in detail.
\end{abstract}

Index Terms-Flag manifold, kinematics singularities, manipulator design, parallel manipulators, stratification.

\section{INTRODUCTION}

$\mathbf{P}$ ARALLEL robots have remarkable advantages over serial robots in terms of load-carrying capacity, stiffness, and accuracy. Nevertheless, the closed-loop nature of parallel robots limits the motion of the platform and creates complex kinematic singularities inside the workspace [21]. In a singular configuration, the mechanism gains some degrees-of-freedom (DOF) and becomes uncontrollable. Furthermore, the actuator forces may become very large which could result in a breakdown of the robot. Therefore, the identification of the singularity loci of parallel robots is a very important design and application issue.

Standard analytic techniques for identifying when a parallel manipulator is at a singularity lead to extremely large equations that are difficult to derive and, therefore, to analyze fully. Note that, for example, the singularity locus of a simple 3-RRR planar parallel manipulator for a constant orientation is the vanishing set of a polynomial of degree 42 [3].

From the design point of view, it is desirable to obtain the analytical expression of the singularity locus of any given

Manuscript received November 10, 2006; revised March 22, 2007. This paper was recommended for publication by Associate Editor I. Bonev and Editor F. Park upon evaluation of the reviewers' comments. The work of F. Thomas and C. Torras was supported in part by the Spanish Ministry of Science and Technology (MCYT) under Project DPI2004-07358 and the Catalan Research Commission through the Robotics group. The work of M. Alberich-Carramiñana was supported in part by DGYCIT MTM2005-01518 and DGYCIT MTM200614234-C02-02 and the Catalan Research Commission.

M. Alberich-Carramiñana is with the Institut de Robòtica i Informàtica Industrial (CSIC-UPC), 08028 Barcelona, Spain, and also with the Department of Applied Mathematics I, UPC, 08028 Barcelona, Spain. (e-mail: maria.alberich@ upc.edu).

F. Thomas and C. Torras are with the Institut de Robòtica i Informàtica Industrial (CSIC-UPC), 08028 Barcelona, Spain (e-mail: torras@iri.upc.edu; thomas @ iri.upc.edu).

Color versions of one or more of the figures in this paper are available online at http://ieeexplore.ieee.org.

Digital Object Identifier 10.1109/TRO.2007.903819 parallel robot to determine the locations of singularities within the given workspace and try to avoid them. This work has been carried out for different particular parallel architectures (see, for example, [8], [9], [14], and [27]). In $1998 \mathrm{Kim}$ and Chung [17] obtained the analytic expression of the singularity locus of the general Gough-Stewart platform with constant orientation using the local structuration method. Later on, Mayer St-Onge and Gosselin [25] found the same result by expanding the Jacobian matrix of the mechanism with constant orientation using linear decomposition and cofactor expansion. Finally, very recently, Li et al. [20] presented an analytic form of the 6-D singularity locus of the general Gough-Stewart platform. The method is based on the cascaded expansion of the determinant of the Jacobian matrix of the mechanism.

The analytical expression for the singularity locus in the 6-D Cartesian space is a polynomial in six variables, the variables of the configuration space of the platform. Hence, the singularity locus is an algebraic set embedded in the configuration space of the platform. As a consequence, this space can be decomposed into connected submanifolds of strictly-diminishing dimensions. This decomposition, together with the adjacency graph between these submanifolds, is technically known as a stratification. The stratification of the configuration space with its embedded singularity locus is much more useful for practical applications than the expression of the unstructured singularity locus itself. This can be seen through an example.

Fig. 1(a) shows a 3-RRR planar parallel manipulator, whose singularity locus for a constant orientation is known to be the vanishing set of a polynomial of degree 42 [3], as displayed in Fig. 1(b). The configuration space of this manipulator can be decomposed into singularity-free 2-D cells, such as $C_{1}$ and $C_{2}$ in Fig. 1(c). The singularity locus defines precisely the boundaries of these 2-D cells. By virtue of the decomposition being a stratification, the boundary of each 2-D cell consists of singular 1-D cells, as for example $C_{3}$, and 0-D cells, as $C_{4}$ and $C_{5}$. The availability of such a well-structured description of the singularity locus permits generating trajectories away from singularities or, if required, crossing singular cells in a controlled way, so that the operative workspace of the parallel manipulator becomes enlarged.

The illustrative example in Fig. 1 includes singularities of both type I and type II [12], whereas in this paper, we are concerned only with type II singularities, which are those of interest when studying the direct kinematics of parallel robots. Unfortunately, with the current tools, deriving the stratification of the singularity locus of the general Gough-Stewart platform is still a fabulous task. However, this task is greatly simplified for the class of platforms known as flagged parallel manipulators, because the stratification of their singularity loci has been shown to have a well-behaved structure inherited from the stratification of the flag manifold [26]. Following this result, in this paper, we 


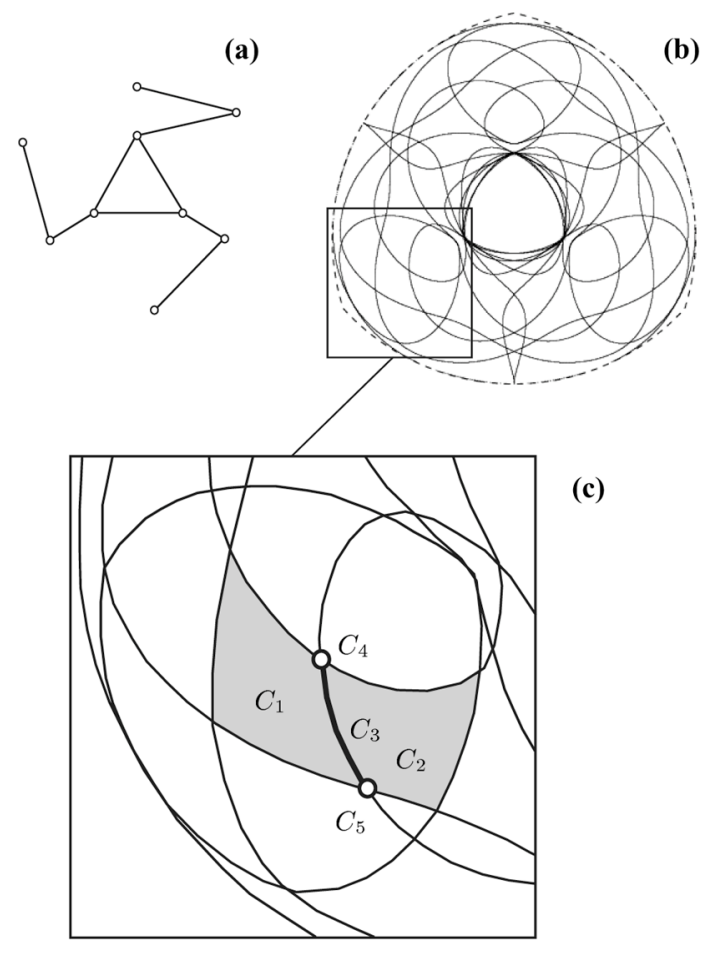

Fig. 1. (a) 3-ㅌRR planar parallel manipulator. (b) It's singularity locus for a constant orientation is the root locus of a polynomial of degree 42 (adapted from [3]). (c) Then, the configuration space of the manipulator can be stratified into cells of strictly diminishing dimension.

characterize in detail the most relevant portion of the stratification of the configuration space (C-space for short) of flagged manipulators. This includes all cells of dimension 6 (nonsingular) and dimension 5 (singular), together with their adjacencies. Previously to that, we assess the interest of carrying out such detailed analysis by expanding the family of flagged manipulators, which turns out to be very large.

This paper is structured as follows. Section II introduces the notion of flag and motivates the interest of flagged manipulators. In Section III, the whole family of flagged manipulators is obtained in a rather intuitive way. Section IV is devoted to the stratification of the configuration space of flagged manipulators, and the topology of the cells of dimension 6 (nonsingular) and dimension 5 (singular) is worked out in detail. Finally, Section V provides some conclusions and points that deserve further attention.

\section{Flags AND FlaGged MANIPULATORS}

Let us consider a plane, a line, and a point so that the point is contained in the line and the line in the plane. This geometric entity is called a flag (see Fig. 2).

A parallel manipulator whose singularities can be described in terms of incidences between two flags adequately placed on its platform and base, respectively, is called a flagged manipulator. As an example, let us take the 3/2 Hunt-Primrose parallel manipulator [15], so called because it has three legs meeting at a platform point and two other legs meeting at a point in the base. Two flags can be placed in its platform and base, as shown in Fig. 3, so that singular configurations correspond to incidences between the elements of the two flags. In Fig. 3, the manipulator

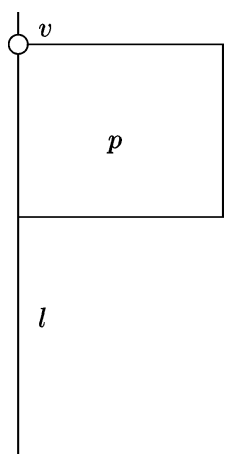

Fig. 2. Flag consists of a point $v$, a line $l$, and a plane $p$, such that $v$ is on $l$ and $l$ is on $p$.

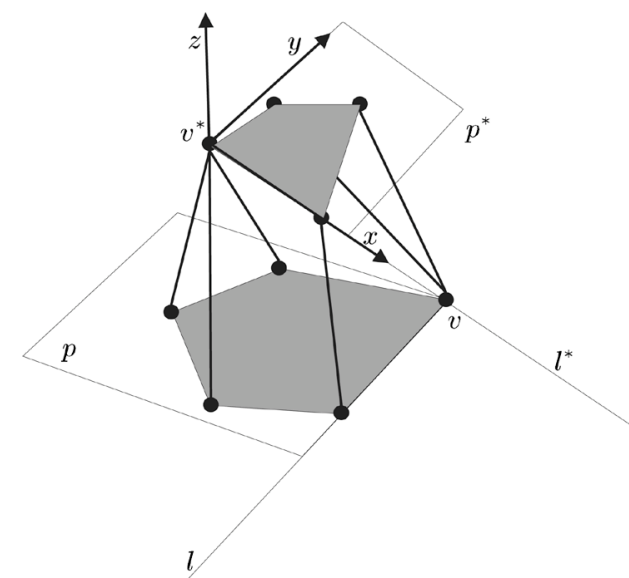

Fig. 3. Singular configuration of a $3 / 2$ Hunt-Primrose manipulator, in which two of the tetrahedra defining its forward kinematics are degenerate.

is shown in a singular configuration characterized by the point $v$ of the base flag lying on the line $l^{*}$ of the platform flag. Besides the $3 / 2$ parallel manipulator and all its specializations, some instances of 3-2-1 manipulators are also known to be flagged [26].

The interest of flagged manipulators derives from the fact that their singularity analysis is quite simple, because:

1) their singularity spaces (consisting of only type II singularities) can be easily decomposed into manifolds, or cells, forming what in algebraic geometry is called a "stratification";

2) each cell can be characterized using a single local chart whose coordinates directly correspond to uncoupled translations and/or rotations in the workspace of the manipulator.

Once these nice properties of flagged manipulators were established [26], our aim in this paper is two-fold. First, we would like to assess the significance of this result by determining the size of the family of manipulators that share these properties. For this purpose, in Section III, we define a transformation of the manipulator legs that leaves singularities invariant. Observe that, for all the members of the resulting family, the topology of their singularity spaces will be the same irrespective of changes in their kinematic parameters.

Second, beyond the purely combinatorial result [26], we would like to derive in detail the previously mentioned topology of singularities so that it becomes operational in practice and 


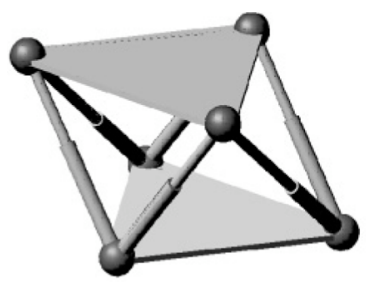

(a)

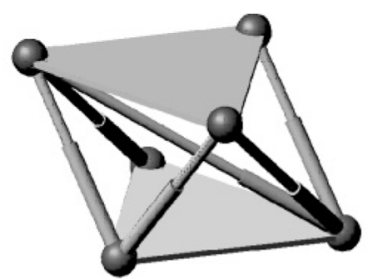

(b)

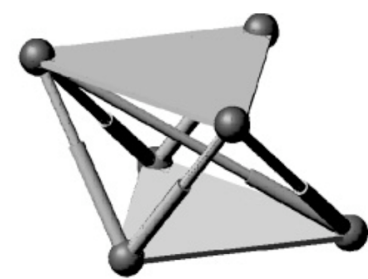

(c)

Fig. 4. Three possible architectures for the 3-3 parallel manipulators.

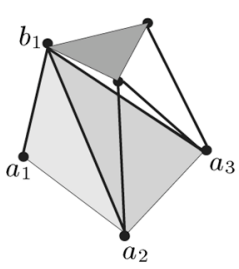

(a)

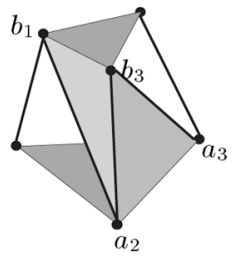

(b)

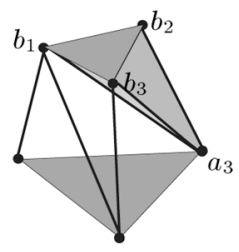

(c)
Fig. 5. Tetrahedra involved in the computation of the forward kinematics of the parallel manipulator in Fig. 4(b).

it permits elucidating questions such as whether any path connecting two assembly modes passes necessarily through a singularity, a seemingly intuitive fact that does not always hold [16].

\section{EXPANDING THE FAMILY OF FLAGGED MANIPULATORS}

Let us consider the set of six-legged manipulators whose leg endpoints merge into three multiple spherical joints both in the base and the platform. There are only three possible architectures for these kind of manipulators, also known as 3-3 manipulators (see Fig. 4). One of them corresponds to the wellknown octahedral manipulator [see Fig. 4(a)], whose forward kinematics is not solvable in closed form [10]. On the contrary, the forward kinematics of the other two can be solved by a sequence of three consecutive trilaterations [5], [15], [24] leading to eight solutions or assembly modes.

Now, let us concentrate our attention on the forward kinematics of the parallel manipulator in Fig. 4(b). Given the lengths of the segments $\overline{a_{1} b_{1}}, \overline{a_{2} b_{1}}$, and $\overline{a_{3} b_{1}}$, there are two possible mirror locations for $b_{1}$ with respect to the plane defined by $a_{1}$, $a_{2}$, and $a_{3}$ [see Fig. 5(a)]. Once one of these two solutions for $b_{1}$ is chosen, $a_{2}, a_{3}, b_{1}$, and $b_{3}$ define another tetrahedron with known edge lengths [see Fig. 5(b)]. Again, there are two possible mirror locations for $b_{3}$, in this case, with respect to the plane defined by $a_{2}, a_{3}$, and $b_{1}$. Finally, after choosing one of the two solutions, $a_{3}, b_{1}, b_{2}$, and $b_{3}$ define another tetrahedron with known edge lengths [see Fig. 5(c)]. In this case, there are two mirror locations for $b_{2}$ with respect to the plane defined by $b_{1}$, $b_{3}$, and $a_{3}$. We conclude that if, and only if, the points in the sets $\left\{a_{1}, a_{2}, a_{3}, b_{1}\right\},\left\{a_{2}, a_{3}, b_{1}, b_{3}\right\}$, and $\left\{a_{3}, b_{1}, b_{2}, b_{3}\right\}$ form nondegenerate tetrahedra, there are eight possible configurations for the moving platform compatible with a given set of leg lengths. Otherwise, the parallel manipulator is in a singularity. Alternatively, we can say that the manipulator is in a singularity if $b_{1}$ is on the base plane, the lines defined by $a_{2} a_{3}$ and $b_{1} b_{3}$ intersect, or $a_{3}$ is on the platform plane. This reinterpretation is important

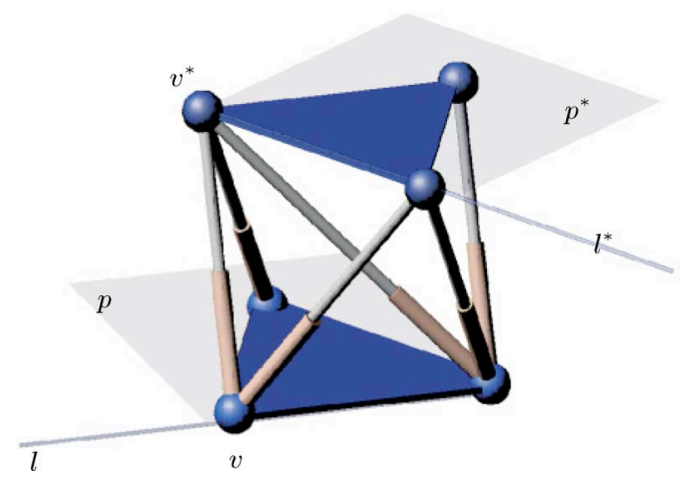

Fig. 6. Basic flagged manipulator and its attached flags.

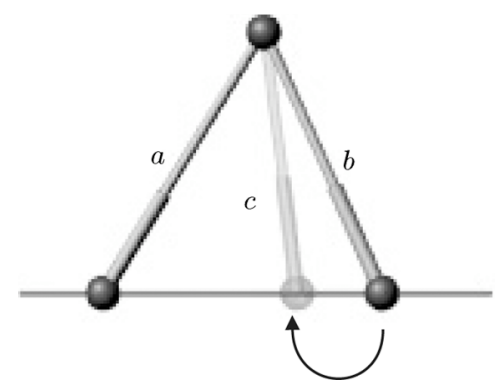

Fig. 7. Simple local transformation that leaves singularities invariant.

because it is not expressed in terms of leg locations but directly in terms of points and edges attached to either the base or the platform. Therefore, if two flags are placed on the manipulator base and platform as shown in Fig. 6, then the manipulator singularities coincide with flag configurations in which either the vertex of one flag lies on the plane of the other flag or the two flag lines intersect.

In what follows, the parallel manipulator in Fig. 6 is called the basic flagged manipulator. Moreover, $v, l$, and $p$ will denote the point, line, and plane of the flag attached to the base, while the same letters with an asterisk will stand for the same flag features of the flag attached to the platform.

\section{A. Family of Six-Legged Flagged Manipulators}

By appropriately moving some leg endpoints of the basic flagged manipulator, other six-legged manipulators having the same singularity locus can be generated. The simple local transformation shown in Fig. 7 serves this purpose. This transformation can be applied to any pair of legs (labelled $a$ and $b$ in Fig. 7) sharing an endpoint, and it consists in moving one of the nonshared endpoints along the line passing through the other 


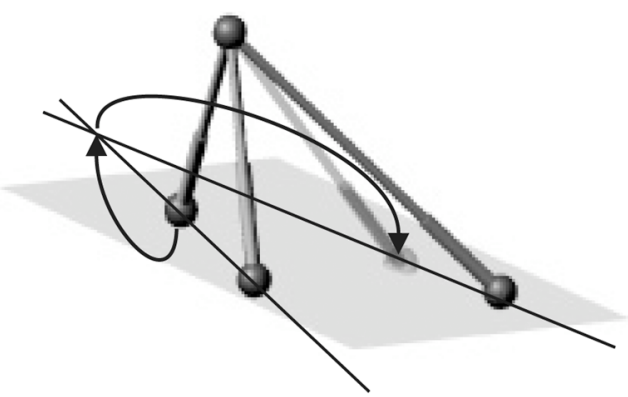

(a)

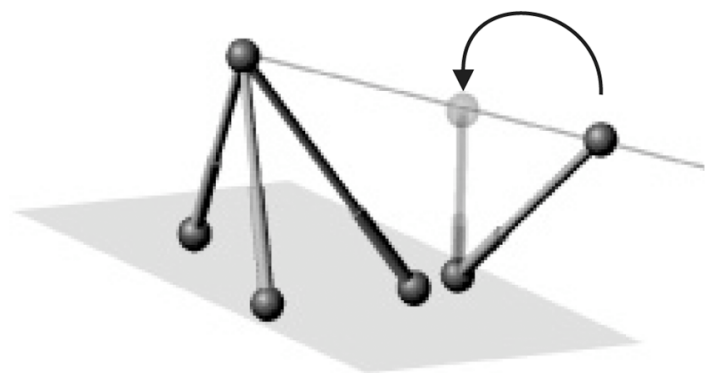

(b)

Fig. 8. Two other local transformations that can be obtained by applying (a) twice and (b) five times the transformation in Fig. 7. The starting configuration in each drawing is that shown with solid legs and the transformation entails moving one leg to the final position depicted as a transparent leg.

nonshared endpoint (as indicated with an arrow in Fig. 7). Of course, the transformation is valid as long as the two nonshared endpoints are not made coincident.

Taking $a$ and $b$ to be the lengths of the two legs in the original pair and $c$ to be the length of the new leg resulting from the transformation, it is clear that there is a one-to-one mapping 1 $g: \mathbb{R}^{2} \rightarrow \mathbb{R}^{2}$ that assigns to each $(a, b)$ a unique $(a, c)$ so that the kinematics of the shared endpoint with respect to the line joining the two nonshared endpoints remains invariant. ${ }^{2}$ Therefore, the forward kinematic model of the entire flagged manipulator is topologically preserved by this local transformation, and thus its singularities of type II remain invariant.

Other more complex transformations can be derived from this simple one. For example, when three legs share an endpoint [see Fig. 8(a)], then any of the three nonshared endpoints can be moved anywhere in the plane by concatenating two simple local transformations. This concatenated transformation maintains the kinematics of the shared endpoint with respect to the plane defined by the three nonshared endpoints. A transformation resulting from concatenating five simple transformations is shown in Fig. 8(b). It consists in moving one of the three endpoints of the tripod to coincide with the endpoint of the leg to be moved, then applying the move marked with an arrow, and, finally, undoing the movement of the tripod.

These transformations permit expanding the whole family of flagged manipulators shown in Fig. 9. As an example, Fig. 10

\footnotetext{
${ }^{1}$ The analytic expression of the $g$ mapping relating the values of $b$ and $c$ as a function of $a$ can be derived from Stewart theorem, named after Matthew Stewart who published it in 1745 .

${ }^{2}$ This point-line kinematic invariance has led the "V"-shaped leg pair to be sometimes denoted a PL (Point-Line) component [18].
}

shows how the 3/2 Hunt-Primrose manipulator [15] can be derived from the basic flagged manipulator by applying a sequence of four of these transformations.

Note that, besides the 3/2 manipulator (leftmost 4-5 instance in Fig. 9), several other members of this family have attracted attention in the past due to their nice properties. The basic 3-3 flagged manipulator was studied by Collins and McCarthy [7], who demonstrated that its singularity locus is a cubic surface that factors into three planes, which can be viewed as the flattening of the three tetrahedra appearing in Fig. 5. In Section IV-C it will become clear that these three planes or flat tetrahedra correspond to the three types of 5-D cells appearing in the stratification of the singularity locus of flagged manipulators.

Zhang and Song [28] analysed a class of six-legged manipulators that had closed-form solution, namely those that had five endpoints aligned in either their platform or their base. Note that all but six of the manipulators appearing in Fig. 9 belong to this class. Among those not belonging to the class are the already mentioned $3 / 2$ manipulator, the $(3-1-1-1)^{2}$ manipulator (third on the left 4-4 instance in Fig. 9) studied by Bruyninckx [4], and the 2-1-1-2/1-1-2-1-1 manipulator (fourth on the left 4-5 instance in Fig. 9), whose potential will be discussed next.

\section{B. Family of Three-Legged Flagged Parallel Manipulators}

Parallel manipulators are characterized by having a base and a platform connected by serial chains. When each of these serial chains is composed of a passive universal joint, a prismatic actuator and a passive spherical joint, the resulting manipulator is called a Gough-Stewart platform. Nevertheless, more general chains can be used between the base and the platform to provide an alternative to prismatic actuators.

The just mentioned 2-1-1-2/1-1-2-1-1 manipulator [see Fig. 12(a)] can be viewed as a 2-2-2 Stewart platform in which one of the two-leg groups has been inverted and a proper alignment of leg-endpoints on the base is maintained. The two-leg groups can be replaced by other equivalent parallel mechanisms [see Fig. 11(b)] or linear chains [see Fig. 11(c)] without altering the singularity locus of the manipulator. Each substitute of a two-leg group should be actuated either by two 1-DOF joints or by one 2-DOFs joint.

As an example, if we substitute each of the three two-leg groups of the 2-1-1-2/1-1-2-1-1 manipulator by a $\underline{P} \underline{P S}$ serial chain [see Fig. 11(c)], the result appears in Fig. 12(b). Collins and Long [6] have considered the replacement of the two-leg groups of a 2-2-2 Stewart platform by kinematically equivalent pantograph mechanisms, such as that in Fig. 11(b).

Notice that the singularities of flagged manipulators considered in this paper are those of type II in the classification of Gosselin and Angeles [12], namely those in which the platform gains one or more DOF and, therefore, cannot resist one or more forces or moments even when all actuators are locked. These are the only ones appearing when prismatic joints are taken to be unlimited. Of course, prismatic actuators have limits which originate singularities of type I, and some manipulator instances with very particular architectural parameters have singularities 


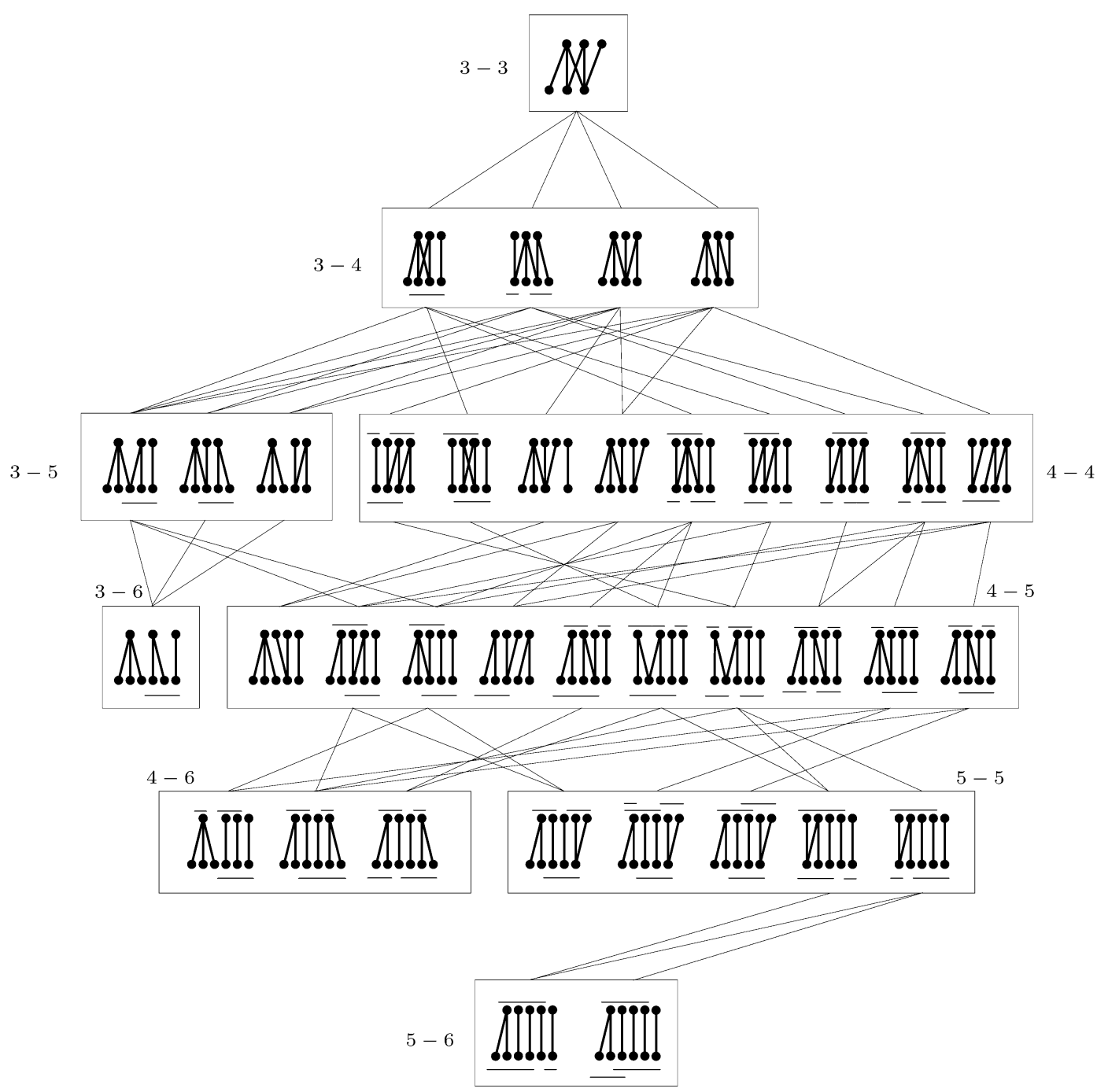

Fig. 9. Whole family of six-legged flagged manipulators expanded from the basic one by applying the transformation in Fig. 8(a). Segments, next to either the base or the platform, indicate the endpoints that should be kept aligned.
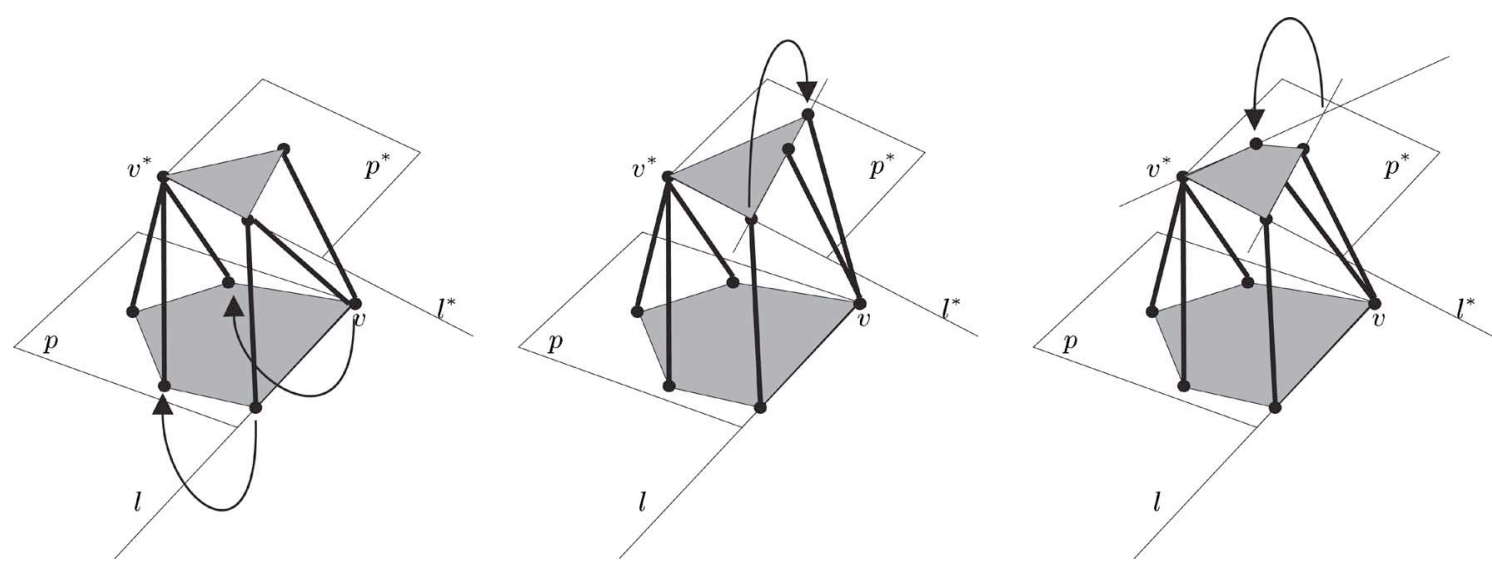

Fig. 10. 3/2 Hunt-Primrose manipulator is a flagged manipulator because it can be obtained by applying a sequence of four local transformations to the basic flagged manipulator. Notice how the attached flags remain invariant under these transformations.

of type III, but these are very specific and well-studied. It is worth mentioning that, by substituting two-leg pairs by other kinematically equivalent mechanisms, the singularities of type II remain unchanged, but new singularities of type I and type
III may be introduced. For instance, the pantograph reaches a singularity of type I when the left and right chains are aligned, even for unlimited actuators. Moreover, if the four links of the pantograph have the same length, then a singularity of type III 


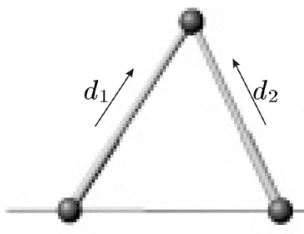

(a)

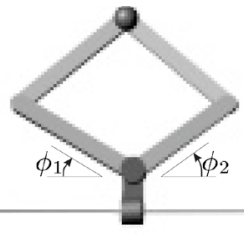

(b)

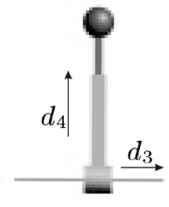

(c)
Fig. 11. (a) Two-leg group can be substituted, for example, by (b) a pantograph or (c) a PRPS serial chain.

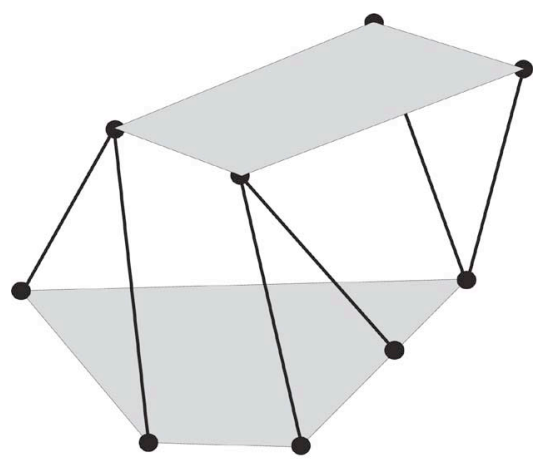

(a)

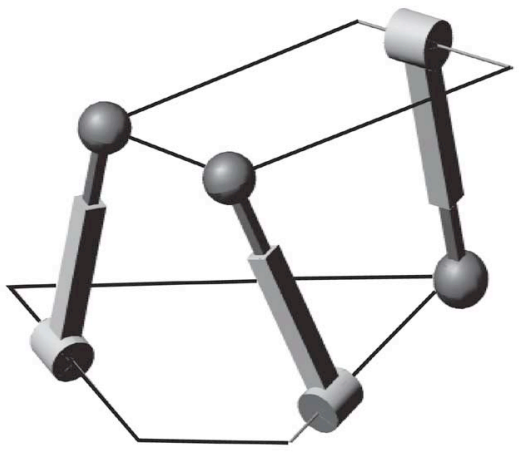

(b)

Fig. 12. (a) 2-1-1-2/1-1-2-1-1 manipulator and (b) a three-legged flagged manipulator derived from it by substituting the three two-leg groups by $\underline{P} \underline{P} S$ serial chains satisfying the required alignment on the base.

appears when the two lower links are aligned. The former corresponds to the workspace boundary, whereas the second is usually a physically unreachable configuration.

Recently, Ben-Horin and Shoham [2] have studied the replacement of the two-leg groups of a 2-2-2 Stewart platform by kinematically equivalent serial chains involving spherical $(\mathrm{S})$, prismatic $(\mathrm{P})$, revolute $(\mathrm{R})$, helical $(\mathrm{H})$, cylindrical $(\mathrm{C})$, and universal (U) joints. By substituting each of the three two-leg groups by the same kinematic chain, they obtained 144 different manipulator architectures. If the combination of different serial chains were allowed, the number of possible manipulator architectures would grow beyond $5 \times 10^{5}$.

Likewise, the family of flagged manipulators displayed in Fig. 9 can be further expanded by replacing two-leg groups with linear chains among the 144 identified by Ben-Horin and Shoham. Just the expansion of the 2-1-1-2/1-1-2-1-1 manipulator leads to a class as large as that developed in [2].

\section{TOPOlOGY OF Singularities OF FLAGGED MANIPULATORS}

Once the significance of the family of flagged manipulators has been established, we will proceed to study their configuration space and, hence, their embedded singular manifolds. The combinatorics of the singularities of flagged manipulators was already made clear in [26], and thus here we will go on to derive the topology of the singularity locus in terms of the 6-D and 5-D cells together with their adjacencies.

To this end, we should now proceed more formally as follows. Section IV-A starts with a brief review of the classical theory of projective flags, which is necessary to contextualize the main result stated in this subsection (and derived in more detail in Appendix B): a stratification of the set parameterizing the affine flags as well as the adjacencies between its strata. Section IV-B shows the connection of the set of affine flags with the configuration space of the manipulator, via a four-fold covering morphism from the latter to the former. This enables, in Section IV-C, to infer the topology of the stratification of the singularity loci of flagged manipulators.

\section{A. From Projective Flags to Affine Flags}

Definition 1 (Flag): A flag in projective space $\mathbb{P}^{3}$ is a sequence $V_{0} \subset V_{1} \subset V_{2} \subset \mathbb{P}^{3}$ of projective subspaces such that $\operatorname{dim}\left(V_{i}\right)=i . V_{0}, V_{1}$, and $V_{2}$ are called the flag features.

The Euclidean space $\mathbb{R}^{3}$ can be viewed as a subspace of $\mathbb{P}^{3}$ via $\mathbb{R}^{3} \cong \mathbb{P}^{3} \backslash \Pi_{\infty}$, where $\Pi_{\infty}$ stands for the plane at infinity. The flags we will be concerned with are the affine flags, that is, flags $V_{0} \subset V_{1} \subset V_{2} \subset \mathbb{P}^{3}$ satisfying $V_{0} \notin \Pi_{\infty}$.

In what follows, we make a slight abuse of language by identifying affine subspaces of dimensions 0,1 , and 2 , in projective space $\mathbb{P}^{3}$ not contained in $\Pi_{\infty}$ with points, lines, and planes, in Euclidean space $\mathbb{R}^{3}$.

Definition 2 (Flag Manifold): The flag manifold $\mathcal{F} \operatorname{lag}(4)$ is the set of all flags in $\mathbb{P}^{3}$. Let $\mathcal{F}_{\mathcal{A}}\left(\mathbb{P}^{3}\right)$ denote the subset of the affine flags in $\mathcal{F} \operatorname{lag}(4)$.

Let $v \subset l \subset p$ be a fixed reference flag. The flag manifold $\mathcal{F}$ lag(4) admits the following stratification (in fact, cell decomposition):

$$
\mathcal{F} \operatorname{lag}(4)=\cup_{w \in \sum_{4}} B^{w}
$$

where $B^{w}$ is the set of all the flags whose flag features have incidence relations with the reference flag determined by the permutation $w \in \sum_{4}$, with $\sum_{4}$ standing for the set of permutations of four elements [13]. As detailed in Appendix A, each cell $B^{w}$ is isomorphic to $\mathbb{R}^{\text {length }(w) 3}$ and hence it is connected. Furthermore, in the stratification (1), two cells of consecutive dimensions are adjacent if and only if there is a single transposition between their associated permutations.

This leads to an algorithmic procedure to derive the graph of cells for the flag manifold, as was displayed in [26]. Fig. 13 shows the cells of dimensions 6 and 5 and their adjacencies. The rectangle represents the 6 -D cell $B^{(4,3,2,1)}$, while the ellipses are the 5-D cells: $B^{(4,3,1,2)}, B^{(3,4,2,1)}$, and $B^{(4,2,3,1)}$. Each 5-D cell is labelled also with $v-p^{*}, p-v^{*}$, and $l \cdot l^{*}$, respectively, which

${ }^{3}$ The length of a permutation $w$ is defined as the number of inversions in $w$, that is, length $(w)=\operatorname{card}\{i<j: w(i)>w(j)\}$. 


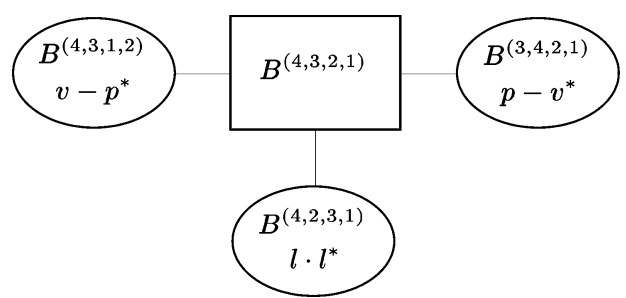

Fig. 13. Stratification of the flag manifold: the rectangle represents the 6-D cell and the ellipses are the 5-D cells.

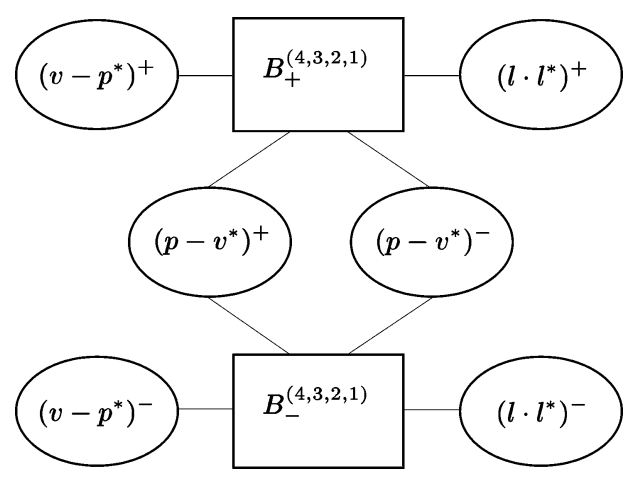

Fig. 14. Stratification of the set of affine flags: the rectangles represent the 6-D cells and the ellipses are the 5-D cells.

characterize the incidence relations between the flag features of the flags $v^{*} \subset l^{*} \subset p^{*}$ in each cell and the reference flag. A hyphen between two elements denotes that one is included in the other and a dot means that they meet at a single point.

The stratification of the flag manifold $\mathcal{F} \operatorname{lag}(4)$ induces a stratification of the subset of affine flags $\mathcal{F}_{\mathcal{A}}\left(\mathbb{P}^{3}\right)$. Indeed, after removing the plane at infinity $\Pi_{\infty}$, the resulting decomposition is still a stratification, and some cells (those whose associated permutations do not start with a 1) are split into two connected components (see Appendix B for details). Fig. 14 shows the cells of dimensions 6 and 5 of $\mathcal{F}_{\mathcal{A}}\left(\mathbb{P}^{3}\right)$ and their adjacencies. The rectangles represents the two 6-D cells $B_{+}^{(4,3,2,1)}$ and $B_{-}^{(4,3,2,1)}$, while the ellipses are the six 5-D cells: $B_{\varepsilon}^{(4,3,1,2)}, B_{\epsilon}^{(3,4, \overline{2}, 1)}$, and $B_{\varepsilon}^{(4,2,3,1)}$, with $\varepsilon \in\{+,-\}$. For the sake of clarity, each 5-D cell is labelled with $\left(v-p^{*}\right)^{\varepsilon},\left(p-v^{*}\right)^{\varepsilon}$, and $\left(l \cdot l^{*}\right)^{\varepsilon}$, respectively, to make explicit the incidence relations between the flag features of the flags in each cell and those of the reference flag.

The stratification of the set of affine flags induces a decomposition of the C-space of flagged manipulators, which we work out in detail in Section IV-B.

\section{B. From Affine Flags to Manipulator Configurations}

Given a flag $\mathcal{V}^{*}=\left(v^{*}, l^{*}, p^{*}\right)$ attached to the basic flagged manipulator as in Fig. 6 (and, in general, to any other member of the family of flagged manipulators), we consider an Euclidean reference frame having $v^{*}$ as origin, $l^{*}$ as the $x$-axis, and $p^{*}$ as the $x-y$ plane. This flag remains invariant when a rotation of $\pi$ radians about any of the three coordinate axes is applied. Formally, the group of Euclidean transformations leaving the flag invariant is $\mathcal{H}_{\mathcal{V}^{*}}=\left\{\mathbf{I}, \mathbf{R}_{x}, \mathbf{R}_{y}, \mathbf{R}_{z}\right\}$, where $\mathbf{I}$ is the identity transformation and $\mathbf{R}_{k}$ stands for a rotation of $\pi$ radians about

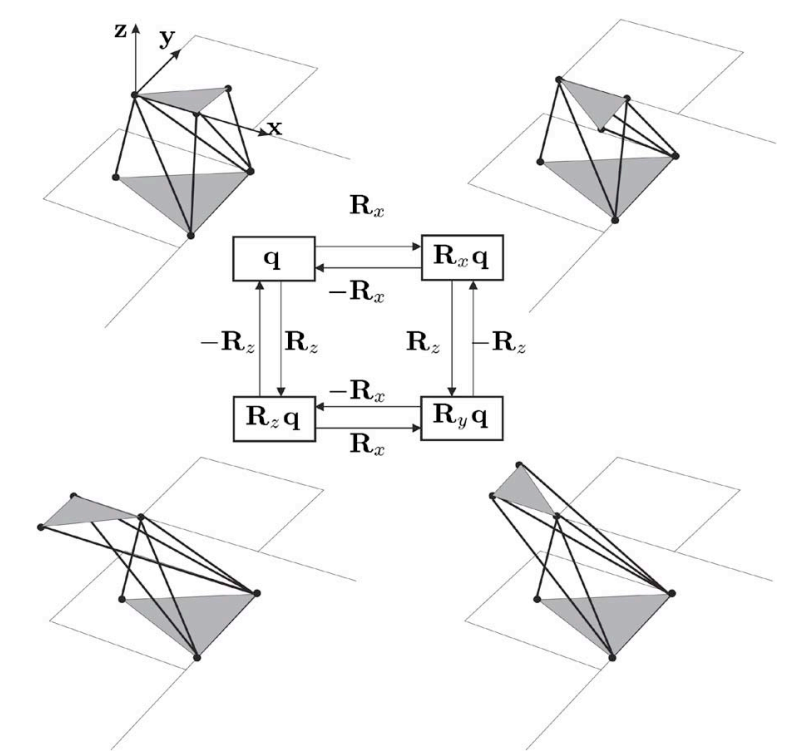

Fig. 15. Four platform configurations sharing the same flag, obtained by rotating $\pi$ radians about its reference axes.

the $k$-axis. Let us mention that $\mathcal{H}_{\mathcal{V} *}$ is one of the representations of the well-known Klein four-group, since $\mathbf{R}_{x} \mathbf{R}_{y}=\mathbf{R}_{y} \mathbf{R}_{x}=$ $\mathbf{R}_{z}, \mathbf{R}_{x} \mathbf{R}_{z}=\mathbf{R}_{z} \mathbf{R}_{x}=\mathbf{R}_{y}$, and $\mathbf{R}_{y} \mathbf{R}_{z}=\mathbf{R}_{z} \mathbf{R}_{y}=\mathbf{R}_{x}$.

Now, let us fix an Euclidean reference frame at the base of the flagged manipulator attached to the reference flag $\mathcal{V}=(v, l, p)$ (and oriented in the same way as the frame previously fixed at the platform). Then, a manipulator configuration is described as $\mathbf{q} \in \mathbb{R}^{3} \times \mathrm{SO}(3)$, relating the platform frame to the base frame. Given one such manipulator configuration $\mathbf{q}$, we can characterize the set of four manipulator configurations yielding this same flag configuration as follows:

$$
\left\{\mathbf{T q} \mid \mathbf{T} \in \mathcal{H}_{\mathcal{V}_{\mathbf{q}}^{*}}\right\}
$$

where $\mathcal{V}_{\mathbf{q}}^{*}$ is the flag associated with the platform in configuration $\mathbf{q}$. This gives a four-fold covering morphism $\pi: \mathbb{R}^{3} \times$ $\mathrm{SO}(3) \rightarrow \mathcal{F}_{\mathcal{A}}\left(\mathbb{P}^{3}\right)$ sending $\mathbf{q}$ to $\mathcal{V}_{\mathbf{q}}^{*}[23]$. Therefore, with each relative configuration of two flags, we can associate four relative configurations of the platform and base.

Fig. 15 shows the four platform configurations sharing the same flag $\mathcal{V}_{\mathbf{q}}^{*}$, namely $\mathbf{q}, \mathbf{R}_{x} \mathbf{q}, \mathbf{R}_{y} \mathbf{q}$, and $\mathbf{R}_{z} \mathbf{q}$.

Summarizing, the configuration space of the manipulator can be seen as a four-fold covering of the set of affine flags. The interesting property is that it thus inherits the nice structure of the latter, as detailed in Section IV-C.

\section{Topology of Singularities}

The covering morphism $\pi$ induces a stratification of $\mathbb{R}^{3} \times \mathrm{SO}(3)$, and hence of the singularity locus of the flagged manipulator, from the stratification of $\mathcal{F}_{\mathcal{A}}\left(\mathbb{P}^{3}\right)$ obtained in Section IV-A. In particular, (2) provides a procedure to unfold the stratification of the affine flags so as to obtain a useful decomposition of the $\mathrm{C}$-space of the manipulator.

Since the covering degree of $\pi$ is 4, the two 6-D disjoint cells in $\mathcal{F}_{\mathcal{A}}\left(\mathbb{P}^{3}\right)$ correspond in $\mathbb{R}^{3} \times \mathrm{SO}(3)$ to eight 6-D cells, that is, eight connected components of the nonsingular manipulator 


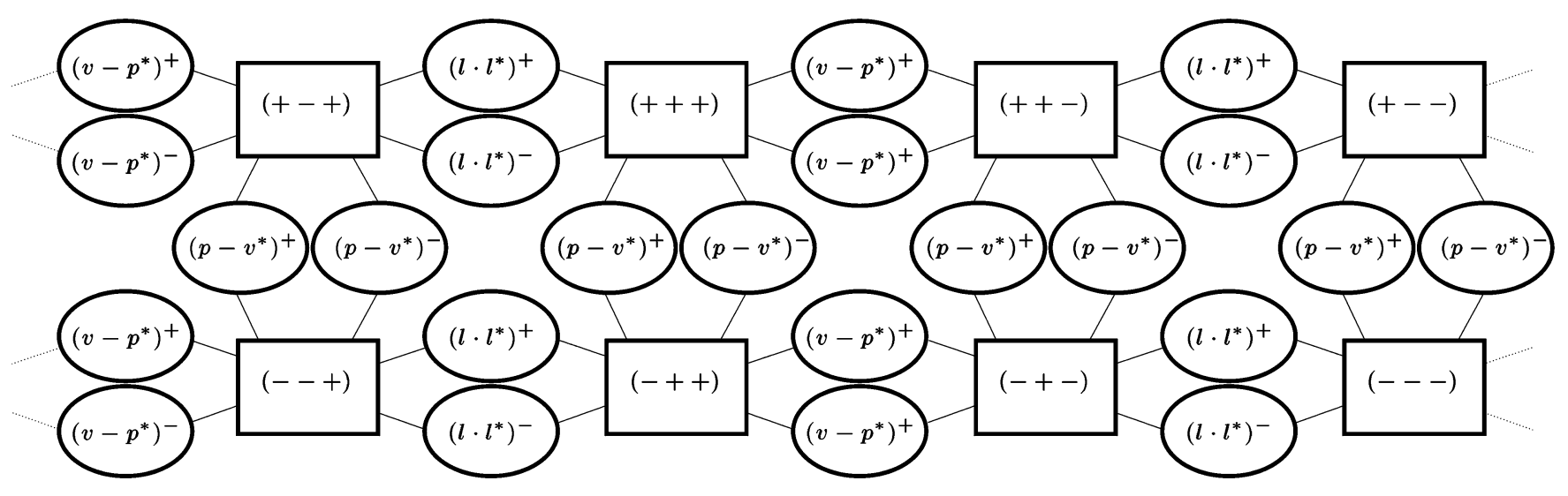

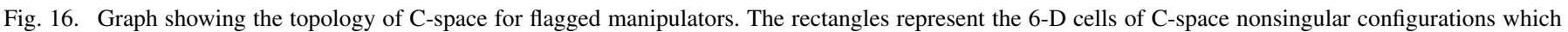
correspond to the eight different assembly modes, while the ellipses are the 5-D cells of singular configurations.

configurations, which (by connectness arguments) must correspond to the eight assembly modes of the flagged manipulators. Hence, there are eight connected components of nonsingular configurations in $\mathrm{C}$-space. To visualize these eight cells see Fig. 15: besides the four platform configurations sharing the same flag $\mathcal{V}_{\mathbf{q}}^{*}$, we could draw the other four configurations corresponding to their images by the specular reflection through the plane of the base.

Now, how are these eight cells packed together in C-space? Owing to the placement chosen for the two flags in the manipulator, the 5-D cells provide a decomposition of the singularity locus.

Recall that there are three 5-D cells in $\mathcal{F l a g}(4)$, which correspond to the cases in which point $v^{*}$ of the platform lies on the base plane, point $v$ of the base lies on the platform plane, and lines $l$ and $l^{*}$ intersect. Restricted to $\mathcal{F}_{\mathcal{A}}\left(\mathbb{P}^{3}\right)$ they split off into six 5-D cells. Since the covering degree of $\pi$ is 4 , this leads in $\mathbb{R}^{3} \times \mathrm{SO}(3)$ to 245 -D cells. We say that a 5-D cell is of type $v^{*}-p, v-p^{*}$, or $l \cdot l^{*}$ if it is one of the connected components of the inverse image of a cell $\left(v^{*}-p\right)^{\varepsilon},\left(v-p^{*}\right)^{\varepsilon}$, or $\left(l \cdot l^{*}\right)^{\varepsilon}$, respectively, for some $\varepsilon \in\{+,-\}$.

As detailed in Appendix C, by resorting to the theory of path lifting [19], the adjacencies between these eight 6-D cells and 24 5-D cells can be derived, resulting in the graph shown in Fig. 16. The rectangles represent the eight 6-D connected components of C-space of nonsingular configurations, while the ellipses are the 5-D manifold patches of singular configurations separating these components. The four multiplicity appears clearly at this level as well. Note that each nonsingular region has the same structure, being bounded by six singular regions, two of type $l \cdot l^{*}$, two of type $v-p^{*}$, and two more of type $p-v^{*}$.

To characterize each 6-D cell we use the triple of signs corresponding to the orientation of the three tetrahedra appearing in Fig. 5, that is, the triple of signs of the determinants of the following ordered set of points (appearing in Fig. 5): $\left\{a_{1}, a_{2}, a_{3}, b_{1}\right\},\left\{a_{2}, a_{3}, b_{1}, b_{3}\right\}$, and $\left\{a_{3}, b_{1}, b_{2}, b_{3}\right\}$. Notice that each pair of 6-D cells differing in only one sign are separated by two different 5-D cells which are both of type $p-v^{*}$, $l \cdot l^{*}$, or $v-p^{*}$, depending on whether the differing sign occupies the first, second, or third position, respectively.
We are now in a suitable position to answer the question raised at the end of Section II. From the graph in Fig. 16, it is clear that any path connecting two assembly modes passes necessarily through a singularity, contrarily to what happens for other types of manipulators [16].

\section{CONCLUSION}

The interest of flagged parallel manipulators has been established from both an intensive and an extensive viewpoint. Concerning the former, we have studied the topology of their configuration space, proving that it admits a well-behaved cell decomposition consisting of eight connected components (cells of dimension 6), corresponding to the eight possible assembly modes, separated by singularities (cells of dimension 5 and lower). The topology of these cells has been formally derived in detail, yielding a structured description of the singularity locus that permits generating trajectories that either avoid or cross singular cells in a controlled way.

To assess the importance of this class of manipulators from an extensive viewpoint, we have studied manipulator transformations that leave singularities invariant. This permits carrying out the singularity analysis on a single representative of this class and the obtained result is guaranteed to be valid for all transformed manipulators. For the sake of simplicity, the representative of the flagged parallel manipulators has been chosen to have a 3-3 architecture. By applying local transformations on its leg endpoint locations, the large family of six-legged flagged manipulators has been expanded. Then, by replacing two-leg groups by kinematically equivalent serial chains, the much larger family of three-legged flagged manipulators has also been derived.

In sum, flagged parallel manipulators have singularity spaces with a well-structured topology, which is the same for all members of this large class, irrespective of changes in their kinematic parameters.

We are currently exploring the possibility of carrying out a similar analysis for the families of manipulators derived from other 3-3 parallel architectures. 


\section{APPENDIX}

A) Stratification of the Flag Manifold Flag(4): Denote by $V_{0} \subset V_{1} \subset V_{2} \subset V_{3}=\mathbb{P}^{3}$ the reference flag. Let $\sum_{4}$ be set of permutations of four elements and consider $w \in \sum_{4}$.

Definition 3 (Bruhat or Schubert Cell): The Bruhat or Schubert cell $B^{w}$ associated to the permutation $w$ is the set of all flags whose flag features have incidence relations with the reference flag determined by $w$ in the following way:

$$
\begin{array}{r}
B^{w}=\left\{\left(V_{0}^{*}, V_{1}^{*}, V_{2}^{*}, V_{3}^{*}\right) \in \mathcal{F} \operatorname{lag}(4): \operatorname{dim}\left(V_{p}^{*} \cap V_{q}\right)=r_{w}(p, q),\right. \\
\text { for } 1 \leq p, q \leq 3\}
\end{array}
$$

where $r_{w}(p, q)=\operatorname{card}\{i \leq p: w(i) \leq q\}-1$.

It is a classical result that each choice of a reference flag gives a stratification or cell decomposition of the flag manifold.

Theorem 1 (Stratification of the Flag Manifold $\mathcal{F}$ lag(4)): (see [13, Ch. 13, Th. 4.3] or [11, Ch. 10]): The disjoint union of all Bruhat cells $B^{w}$ with $w \in \sum_{4}$ is a stratification for $\mathcal{F} \operatorname{lag}(4)$

$$
\mathcal{F} \operatorname{lag}(4)=\cup_{w \in \sum_{4}} B^{w}
$$

and $B^{u} \cap \overline{B^{w}} \neq \emptyset$ if and only if $B^{u} \subset \overline{B^{w}}$.

The structure of each cell and the adjacencies between them are also classically well established.

Proposition 1: (see [13, Ch. 13, Prop. 4.7] or [11, Ch. 10, Prop. 7]):

1) $B^{w}$ is isomorphic to the affine space $\mathbb{R}^{\operatorname{length}(w)}$.

2) If $B^{w}$ and $B^{u}$ are two cells of consecutive dimensions length $(w)=\operatorname{length}(u)+1$, then $\overline{B^{w}} \supset B^{u}$ if and only if there exists a transposition $t \in \sum_{4}$ so that $w=t u$.

For example, if we take $w_{1}=(4,2,3,1), w_{2}=(2,1,4,3)$, and $w_{3}=(3,4,1,2)$, then $B^{w_{1}}, B^{w_{2}}$, and $B^{w_{3}}$ have dimensions 5, 2, and 4, respectively; $B^{w_{1}}$ and $B^{w_{2}}$ are adjacent (i.e., $\left.\overline{B^{w_{1}}} \supset B^{w_{2}}\right)$, while $B^{w_{1}}$ and $B^{w_{3}}$ are not.

B) Stratification of the Set of Affine Flags $\mathcal{F}_{\mathcal{A}}\left(\mathbb{P}^{3}\right)$ : Fix from now on an affine reference flag, that is, $V_{0} \subset V_{1} \subset V_{2} \subset$ $V_{3}=\mathbb{P}^{3}$ with $V_{0} \notin \Pi_{\infty}$. Consider the corresponding cell decomposition of $\mathcal{F}$ lag (4) as in (3). When restricted to the open subset of the affine flags $\mathcal{F}_{\mathcal{A}}\left(\mathbb{P}^{3}\right)$ the partition (3) clearly induces a partition

$$
\mathcal{F}_{\mathcal{A}}\left(\mathbb{P}^{3}\right)=\cup_{w \in \sum_{4}}\left(B^{w} \cap \mathcal{F}_{\mathcal{A}}\left(\mathbb{P}^{3}\right)\right) .
$$

Since the reference flag is an affine flag, none of the previous intersections is empty. However, it might happen that some cell $B^{w}$ would split off into two connected components: indeed, $B^{w} \cap \mathcal{F}_{\mathcal{A}}\left(\mathbb{P}^{3}\right)$ is a unique connected component if and only if the permutation $w$ starts with $w(1)=1$. To see this, choose an affine reference frame $\left\{V_{0} ; \mathbf{e}_{1}, \ldots, \mathbf{e}_{n}\right\}$ attached to the reference flag, namely $\mathbf{e}_{1}$ is a vector representing the improper point $\mathbf{e}_{1}^{\infty}=V_{1} \cap H_{\infty}, \mathbf{e}_{2}$ is a vector representing another point $\mathbf{e}_{2}^{\infty}$ on the improper line $V_{2} \cap H_{\infty}$, and so on. Let $\left(x_{1}, \ldots, x_{n}\right)$ denote the projective coordinates in its associated projective reference $\left\{V_{0}, \mathbf{e}_{1}^{\infty}, \ldots, \mathbf{e}_{n}^{\infty} ; \mathbf{a}\right\}$.

First, let us give a construction of the isomorphism of Proposition 1. Observe that each flag $\mathcal{V}^{*}=\left(V_{0}^{*}, V_{1}^{*}, V_{2}^{*}\right) \in B^{w}$ is represented by a unique $4 \times 4$ matrix $M$ whose first three rows span the flag features of $\mathcal{V}^{*}$, and where the $p$ th row has a 1 in the $w(p)$ th column, with all 0's at the right and below of this 1 .
M will be called the canonical matrix representing the flag $\mathcal{V}^{*}$. For example, for $w=(3,4,2,1)$ the cell $B^{w}$ is isomorphic to the set of matrices of the form

$$
\left(\begin{array}{llll}
* & * & 1 & 0 \\
* & * & 0 & 1 \\
* & 1 & 0 & 0 \\
1 & 0 & 0 & 0
\end{array}\right)
$$

where the stars denote arbitrary real numbers; in this case $B^{w}$ is the set of all flags whose vertex lies on the plane $V_{2}:\left\{x_{4}=\right.$ $0\}$. The number of stars appearing in the canonical matrices parameterizing the flags of $B^{w}$ (for an arbitrary $w$ ) turns out to be the length of $w$ (see $[11,10.2]$ ).

If we switch to affine flags and we take up again the example of the permutation $w=(3,4,2,1)$, the affine flags of $B^{w}$ are the disjoint union of two cells: one of them is isomorphic to the set of matrices of the form

$$
\left(\begin{array}{llll}
a & * & 1 & 0 \\
* & * & 0 & 1 \\
* & 1 & 0 & 0 \\
1 & 0 & 0 & 0
\end{array}\right)
$$

where the stars denote arbitrary real numbers and $a$ denotes a positive real number; the other cell is isomorphic to the set of matrices of the same form (5), where the stars denote arbitrary real numbers and $a$ denotes a negative real number. The matrices of the form (5), where $a$ is zero correspond to flags which are not affine.

For a permutation $w$ with $w(1)>1$, let $B_{+}^{w}$ denote the connected component of $B^{w} \cap \mathcal{F}_{\mathcal{A}}\left(\mathbb{P}^{3}\right)$ formed from the flags $\left\{\left(v^{*}, l^{*}, p^{*}\right) \in B^{w}: v^{*}=\left(x_{1}, x_{2}, x_{3}, x_{4}\right)\right.$ with $\left.x_{1} x_{w(1)}>0\right\}$ and let $B_{-}^{w}$ equal $\left\{\left(v^{*}, l^{*}, p^{*}\right) \in B^{w}: v^{*}=\left(x_{1}, x_{2}, x_{3}, x_{4}\right)\right.$ with $\left.x_{1} x_{w(1)}<0\right\}$. Observe that the quotient $\left(x_{1} / x_{w(1)}\right)$ is the $(1,1)$ entry of the canonical matrix of any flag belonging to $B^{w}$. If $w(1)=1$, set $B_{+}^{w}=B_{-}^{w}=B^{w}$.

The interesting point of partition (4) is that it provides a stratification of $\mathcal{F}_{\mathcal{A}}\left(\mathbb{P}^{3}\right)$ and that the adjacencies between the cells may also be determined:

Theorem 2:

1) The partition

$$
\mathcal{F}_{\mathcal{A}}\left(\mathbb{P}^{3}\right)=\bigcup_{\substack{w \in \sum_{4} \\ w(1) \neq 1}}\left(B_{+}^{w} \cup B_{-}^{w}\right) \cup \bigcup_{\substack{w \in \sum_{4} \\ w(1)=1}} B^{w}
$$

is a stratification for the affine flags.

2) Let $u$ and $w$ be two permutations of $\sum_{4}$

(a) If $B^{u} \subseteq \overline{B^{w}}$, then $B_{+}^{u} \subseteq \overline{B_{+}^{w}}$ and $B_{-}^{u} \subseteq \overline{B_{-}^{w}}$.

(b) If $B^{u} \subseteq \overline{B^{w}}$ and $u(1)<w(1)$, then $B_{+}^{u} \subseteq \overline{B_{-}^{w}}$ and $B_{-}^{u} \subseteq \overline{B_{+}^{w}}$.

Moreover, there are no other adjacencies between cells than those in the previous two cases.

Proof: The proof follows after a deeper insight into the canonical matrices associated to the flags (for details see [1]).

C) Stratification of Configuration Space $\mathbb{R}^{3} \times \mathrm{SO}(3)$ : Via the four-fold covering morphism $\pi: \mathbb{R}^{3} \times \mathrm{SO}(3) \rightarrow \mathcal{F}_{\mathcal{A}}\left(\mathbb{P}^{3}\right)$, introduced in Section IV-B, the stratification of $\mathcal{F}_{\mathcal{A}}\left(\mathbb{P}^{3}\right)$ induces a stratification of $\mathbb{R}^{3} \times \mathrm{SO}(3)$. We shall focus on the cells of 
dimensions 5 and 6 and in determining their adjacencies. To this aim, we need to introduce some concepts and results on paths and path lifting.

Definition 4: A path in a manifold $S$ is a continuous map $\gamma$ from the unit real interval $[0,1]$ to $S ; \gamma(0)$ and $\gamma(1)$ are called the origin and end, respectively, of $\gamma ; \gamma$ is also called transition between $\gamma(0)$ and $\gamma(1)$. The path is closed if $\gamma(0)=\gamma(1)$. The inverse path of $\gamma$ is defined as $\gamma^{-1}(t)=\gamma(1-t)$.

Given a covering morphism $\pi: \widetilde{S} \rightarrow S$, a lift of the path $\gamma:[0,1] \rightarrow S$ is a path on $\widetilde{S}, \widetilde{\gamma}:[0,1] \rightarrow \widetilde{S}$, so that $\pi \circ \widetilde{\gamma}=\gamma$.

Theorem 3 (Uniqueness of the Lifting; See [19, 17.6]): Let $\pi: \widetilde{S} \rightarrow S$ be a covering morphism. Given a path $\gamma:[0,1] \rightarrow S$ and a point $x \in \widetilde{S}$ so that $\pi(x)=\gamma(0)$, there is a unique lift $\widetilde{\gamma}$ of the path $\gamma$ so that $\widetilde{\gamma}(0)=x$.

Recall that to characterize each of the eight 6-D cells of $\mathbb{R}^{3} \times$ $\mathrm{SO}(3)$, we use the triple of signs corresponding to the orientation of the three tetrahedra appearing in Fig. 5. Observe that the four 6-D cells $(\varepsilon,+,+),(\varepsilon,+,-),(\varepsilon,-,+)$, and $(\varepsilon,-,-)$ map by the covering $\pi$ to $B_{\varepsilon}^{(4,3,2,1)}$ for $\varepsilon \in\{+,-\}$.

Theorem 4: Each pair of 6-D cells of $\mathbb{R}^{3} \times \mathrm{SO}(3)$ differing in only one sign are separated by two different 5-D cells which are both of type $p-v^{*}, l \cdot l^{*}$, or $v-p^{*}$, if the differing sign occupies the first, second, or third position, respectively.

Proof: Directly due to the four-fold covering $\pi$, there are two different 5-D cells of type $p-v^{*}$ separating each pair of 6-D cells $\left(+, \varepsilon_{1}, \varepsilon_{2}\right)$ and $\left(-, \varepsilon_{1}, \varepsilon_{2}\right)$ for any $\varepsilon_{1}, \varepsilon_{2} \in\{+,-\}$. Fix a flag $\mathcal{V}^{*}=\mathbf{q}(\mathcal{V}) \in B_{\varepsilon}^{(4,3,2,1)}$, with $\varepsilon \in\{+,-\}$. We will consider in $\mathcal{F}_{\mathcal{A}}\left(\mathbb{P}^{3}\right)$ four different paths with origin $\mathcal{V}^{*}$ that will lie entirely in $B_{\varepsilon}^{(4,3,2,1)}$ except at a point, at which a 5-D cell will be crossed. Namely, $\rho_{x}$ and $\rho_{z}$ are the rotations from 0 to $\pi$ radians about the $x$-axis and $z$-axis, respectively, of a orthogonal reference frame attached to the flag $\mathcal{V}^{*} ; \rho_{x}^{-1}$ and $\rho_{z}^{-1}$ are the respective inverse paths, i.e., rotations from 0 to $-\pi$ radians. Observe that the path $\rho_{x}(t)=\left(v^{*}, l^{*}, p^{*}(t)\right)$ crosses the 5-D cell $\left(v-p^{*}\right)^{\varepsilon}$ at the point $\rho_{x}\left(t_{0}\right)=\left(v^{*}, l^{*}, p^{*}\left(t_{0}\right)\right)$ at which the platform plane $p^{*}\left(t_{0}\right)$ touches the vertex $v$ of the base plane, and that $\rho_{z}(t)=\left(v^{*}, l^{*}(t), p^{*}\right)$ crosses the 5-D cell $\left(l \cdot l^{*}\right)^{\varepsilon}$ at the point $\rho_{z}\left(t_{1}\right)=\left(v^{*}, l^{*}\left(t_{1}\right), p^{*}\right)$ at which the platform line $l\left(t_{1}\right)$ goes through the point $p^{*} \cap l$.

Let $\left\{\mathbf{q}, \mathbf{R}_{x} \mathbf{q}, \mathbf{R}_{y} \mathbf{q}, \mathbf{R}_{z} \mathbf{q}\right\}$ be the four points in the fiber of $\mathcal{V}^{*}=\mathbf{q}(\mathcal{V})$. Consider the lifts of the paths $\rho_{x}, \rho_{x}^{-1}, \rho_{z}$, and $\rho_{z}^{-1}$ with origin q (cf. Theorem 3): $\widetilde{\rho_{x}}, \widetilde{\rho_{x}^{-1}}, \widetilde{\rho_{z}}$, and $\widetilde{\rho_{z}^{-1}}$. Notice that the transitions $\widetilde{\rho_{x}}$ and $\widetilde{\rho_{x}^{-1}}$ do not intersect except at the ends; the different configurations $\mathbf{q}_{t_{0}}$ and $\mathbf{q}_{t_{0}}^{\prime}$ at which $\widetilde{\rho_{x}}$ and $\widetilde{\rho_{x}^{-1}}$, respectively, cross a 5-D cell share the same flag $\rho_{x}\left(t_{0}\right)$, that is, $\pi\left(\mathbf{q}_{t_{0}}\right)=\pi\left(\mathbf{q}_{t_{0}}^{\prime}\right)=\rho_{x}\left(t_{0}\right)$; at $\mathbf{q}_{t_{0}}$ and $\mathbf{q}_{t_{0}}^{\prime}$ the volume of the last tetrahedra appearing in Fig. 5 becomes zero. Hence, each transition crosses a different 5- $\mathrm{D}$ cell in $\mathbb{R}^{3} \times \mathrm{SO}(3)$ of type $v-$ $p^{*}$ and both transitions join two 6-D cells whose differing sign occupies the third position. An analogous reasoning applies for transitions $\widetilde{\rho_{z}}$ and $\rho_{z}^{-1}$ : each of them crosses a different 5-D cell in $\mathbb{R}^{3} \times \mathrm{SO}(3)$ of type $l \cdot l^{*}$ and both transitions join two 6-D cells whose differing sign occupies the second position. Finally, a similar reasoning can be carried out with the lifts of the paths $\rho_{x}$ and $\rho_{x}^{-1}$ with origin $\mathbf{R}_{z} \mathbf{q}$, and with the lifts of the paths $\rho_{z}$ and $\rho_{z}^{-1}$ with origin $\mathbf{R}_{x} \mathbf{q}$ proving, thus completely, the statement of the Theorem and the adjacencies displayed in Fig. 16.

\section{REFERENCES}

[1] M. Alberich-Carramiñana, V. González, and C. Torras, "The topology of singularities of flagged parallel manipulators," IRI Tech. Rep., 2005. [Online]. Available: http://www-iri.upc.es/people/torras/articles/IRIRep05.pdf

[2] P. Ben-Horin and M. Shoham, "Singularity condition of six degree-offreedom three-legged parallel robots based on Grassmann-Cayley algebra," IEEE Trans. Robot., vol. 22, no. 4, pp. 577-590, Aug. 2006.

[3] I. A. Bonev and C. M. Gosselin, "Singularity loci of planar parallel manipulators with revolute joints," in Proc. 2nd Workshop Comput. Kinemat., 2001, pp. 291-299.

[4] H. Bruyninckx, "Closed-form forward position kinematics for a (3 $1-1-1)^{2}$ fully parallel manipulator," IEEE Trans. Robot. Autom., vol. 14, no. 2, pp. 326-328, Apr. 1998.

[5] H. Bruyninckx, "Forward kinematics for Hunt-Primrose parallel manipulators," Mechanism Mach. Theory, vol. 34, pp. 657-664, 1999.

[6] C. L. Collins and G. L. Long, "The singularity analysis of an in-parallel hand controller for force-reflected teleoperation," IEEE Trans. Robot. Autom., vol. 11, no. 5, pp. 661-669, Oct. 1995.

[7] C. L. Collins and J. M. McCarthy, "The singularity loci of two triangular parallel manipulators, proc. of the," in Proc. IEEE Int. Conf. Adv. Robot., 1997, pp. 473-479.

[8] R. DiGregorio, "Analytic formulation of the 6-3 fully-parallel manipulator's singularity determination," Robotica, vol. 19, pp. 663-667, 2001

[9] R. DiGregorio, "Singularity-locus expression of a class of parallel mechanism," Robotica, vol. 20, pp. 323-328, 2002.

[10] D. M. Downing, A. E. Samuel, and K. H. Hunt, "Identification of the special configurations of the octahedral manipulator using the pure condition," Int. J. Robot. Res., vol. 21, no. 2, pp. 147-160, 2002.

[11] W. Fulton, "Young Tableaux," in London Mathematical Society Student Texts. Cambridge, U.K.: Cambridge Univ. Press, 1997, vol. 35.

[12] C. Gosselin and J. Angeles, "Singularity analysis of closed-loop kinematic chains," IEEE Trans. Robot. Autom., vol. 6, no. 3, pp. 281-290, Jun. 1990.

[13] H. Hiller, Geometry of Coxeter Groups, Research Notes in Mathematics. London, U.K.: Pitman, 1982.

[14] Z. Huang, L. H. Chen, and Y. W. Li, "The singularity principle and property of stewart parallel manipulator," J. Robotic Syst., vol. 20, no. 4, pp. 163-176, 2003.

[15] K. H. Hunt and E. J. F. Primrose, "Assembly configurations of some in-parallel-actuated manipulators," Mechanism Mach. Theory, vol. 28, no. 1, pp. 31-42, 1993.

[16] C. Innocenti and V. Parenti-Castelli, "Singularity-free evolution from one configuration to another in serial and fully-parallel manipulators," in Proc. ASME 22nd Biennial Mechan. Conf.: Robot., Spatial Mechan., Mechan. Syst., 1992, pp. 553-560.

[17] D. Kim and W. Y. Chung, "Analytic singularity equation and analysis of six-DOF parallel manipulators using local structuration method," IEEE Trans. Robot. Autom., vol. 5, no. 4, pp. 612-622, Aug. 1999.

[18] X. Kong and C. M. Gosselin, "Generation and forward displacement analysis of two new classes of analytic 6-SPS parallel manipulators," J. Robot. Syst., vol. 18, no. 6, pp. 295-304, 2001.

[19] C. Kosniowski, A First Course in Algebraic Topology. Cambridge, U.K.: Cambridge Univ. Press, 1980.

[20] H. Li, C. M. Gosselin, M. J. Richard, and B. M. St-Onge, "Analytic form of the six-dimensional singularity locus of the general GoughStewart platform," J. Mechan. Des., vol. 128, no. 1, pp. 279-287, 2006.

[21] J.-P. Merlet, Parallel Robots. New York: Springer, 2000.

[22] A. S. Posamenteier and C. T. Salkind, Challenging Problems in Geometry. New York: MacMillan Company, 1970.

[23] P. Sankaran and P. Zvengrowski, "Stable parallelizability of partially oriented flag manifolds II," Can. J. Math, vol. 49, no. 6, pp. 1323-1339, 1997.

[24] S.-K. Song and D.-S. Kwon, "A tetrahedron approach for a unique closed-form solution of the forward kinematics of six-DOF parallel mechanisms with multiconnected joints," J. Robot. Syst., vol. 19, no. 6, pp. 269-281, 2002.

[25] B. M. St-Onge and C. M. Gosselin, "Singularity analysis and representation of the general Gough-Stewart platform," Int. J. Robot. Res., vol. 19 , no. 3, pp. 271-288, 2000.

[26] C. Torras, F. Thomas, and M. Alberich-Carramiñana, "Stratifying the singularity loci of a class of parallel manipulators," IEEE Trans. Robot., vol. 22, no. 1, pp. 23-32, Feb. 2006.

[27] A. Wolf and M. Shoham, "Investigation of parallel manipulators using linear complex approximation," ASME Trans. J. Mechan. Des., vol. 125 , pp. 564-572, 2003

[28] C. Zhang and S.-M. Song, "Forward kinematics of a class of parallel (Stewart) platforms with closed-form solutions," in Proc. Int. Conf. Robot. Autom., 1991, pp. 2676-2681. 


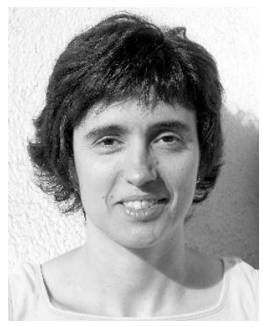

and computer vision.

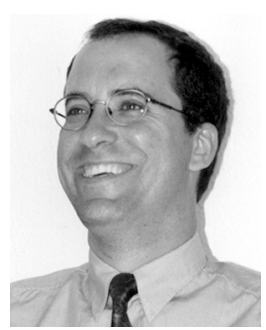

Federico Thomas (M'06) received the telecommunications engineering degree and the Ph.D. degree (with honors) in computer science from the Technical University of Catalonia (UPC), Barcelona, Spain, in 1984 and 1988, respectively.

$\mathrm{He}$ is a Research Professor with the Spanish Scientific Research Council (CSIC), Barcelona, Spain. In 1999, he was Visiting Professor, sponsored by the Autonomous Government of Catalonia, with the Computing Laboratory, Oxford University, London, U.K. He has been a Project Leader of several national projects financed by the Spanish Committee for Science and Technology (CICYT) and by local companies such as ENHER, a power generation company now part of ENDESA. His current research interests include geometry and kinematics with applications to robotics, computer graphics, and computer Vision. He is director of the Institut de Robòtica i Informàtica Industrial (CSIC-UPC).

Dr. Thomas is an Associate Editor of the IEEE TRANSACTIONS ON ROBOTICS.

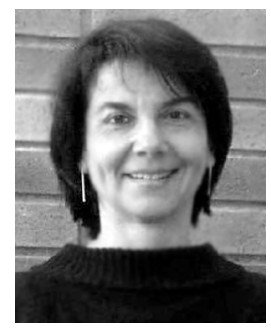

Carme Torras (M'07) received the M.Sc. degree in mathematics from the Universitat de Barcelona, Barcelona, Spain, the M.Sc. degree in computer science from the University of Massachusetts, Amherst, and the Ph.D. degree in computer science from the Technical University of Catalonia (UPC), Barcelona, Spain.

She is a Research Professor with the Spanish Scientific Research Council (CSIC), Barcelona, Spain. She has published four books and more than 100 papers in the areas of robot kinematics, geometric reasoning, computer vision, and neurocomputing. She has been a local Project Leader of several European projects, such as "Planning RObot Motion" (PROMotion), "Robot Control based on Neural Network Systems" (CONNY), "Self-organization and Analogical Modelling using Subsymbolic Computing" (SUBSYM), "Behavioural Learning: Sensing and Acting" (B-LEARN), and the ongoing 6th framework IP project "Perception, Action and COgnition through Learning of Object-Action Complexes" (PACO-PLUS). 